OPEN ACCESS

Edited by:

Zongbing You,

Tulane University, United States

Reviewed by:

Daotai Nie,

Southern Illinois University School of

Medicine, United States

Li Liu,

UT Southwestern Medical Center,

United States

*Correspondence:

Xiawei Wei

xiaweiwei@scu.edu.cn

${ }^{\dagger}$ These authors have contributed equally to this work

Specialty section:

This article was submitted to

Surgical Oncology,

a section of the journa

Frontiers in Oncology

Received: 12 April 2019

Accepted: 19 August 2019

Published: 10 September 2019

Citation:

Ma X, Wang M, Yin T, Zhao Y and

Wei X (2019) Myeloid-Derived

Suppressor Cells Promote Metastasis

in Breast Cancer After the Stress of

Operative Removal of the Primary

Cancer. Front. Oncol. 9:855.

doi: 10.3389/fonc.2019.00855

\section{Myeloid-Derived Suppressor Cells Promote Metastasis in Breast Cancer After the Stress of Operative Removal of the Primary Cancer}

\author{
Xuelei Ma ${ }^{1 \dagger}$, Manni Wang ${ }^{1 \dagger}$, Tao Yin ${ }^{1}$, Yunuo Zhao ${ }^{1}$ and Xiawei Wei ${ }^{2 *}$ \\ 'State Key Laboratory of Biotherapy, Department of Biotherapy, Cancer Center, West China Hospital, Sichuan University, \\ Chengdu, China, ${ }^{2}$ Lab of Aging Research and Nanotoxicology, State Key Laboratory of Biotherapy, West China Hospital, \\ Sichuan University, Chengdu, China
}

Objective: To investigate the role of myeloid-derived suppressor cells (MDSC) in cancer progression after the stress of operative removal and the potential treatment value of MDSC depletion.

Summary Background Data: Surgery is the most important treatment strategy in breast cancer. Recent research has provided evidence that operations may promote cancer metastases under some circumstances.

Methods: A mouse model of breast cancer (administration of the murine breast cancer 4T1 cells subcutaneously) and the stress of operation were used to compare immune responses and survival outcomes. Flow cytometry was performed to detect the expression of CD11b and Gr1 MDSCs in tumor tissues and lung metastases. Cytokine levels were detected with three-color flow cytometry and enzyme-linked immunosorbent assay (ELISA). MDSCs were isolated and co-cultured with 4T1 cells to identify any morphological change with immunofluorescence. The anti Gr-1 antibody was used to detect the function of the anti-Gr1 treatment in breast cancer.

Results: The operative stress impaired the overall survival, leading to an increased number of MDSCs that preferentially infiltrated the tumor microenvironment and promoted tumor metastasis. In both in vitro and in vivo assays, MDSCs induced the epithelial-mesenchymal transition (EMT) of tumor cells through the up-regulation of TGF-beta1, VEGF, and IL-10. Furthermore, a treatment strategy of MDSC depletion was found to reduce pulmonary metastases after operations.

Conclusions: The stress of operation could impair the overall survival in mice. The infiltrated MDSCs appear to induce EMT of tumor cells and increase metastases through the up-regulation of TGF-beta1, VEGF, and IL-10 levels. MDSC depletion could be a promising treatment strategy to prevent immune evasion after operations.

Keywords: MDSC (myeloid-derived suppressor cells), operation, stress, breast cancer, metastasis 


\section{INTRODUCTION}

Breast cancer is one of the most common malignancies worldwide for women (1). More than 1,380,000 cases of breast cancer are diagnosed each year with over 450,000 deaths related to breast cancer, among which cancer metastases account directly for over $80 \%$ of these deaths $(2,3)$. In recent decades, we have witnessed various means of treating breast cancer, including operative removal, radiotherapy, chemotherapy, and endocrine therapy. In order to control local complications and improve the quality of life, the majority of patients with breast cancer, especially those with large, symptomatic lesions, now receive a comprehensive, surgery-based therapy $(4,5)$. Several experimental studies, however, have revealed the dual role on operative stress which appears to promote cancer metastasis under certain pathologic conditions (6).

The tumor environment has been reported to play a key role in promoting metastases in patients with solid tumors (7). The operative removal of tumors increases the release of certain cytokines in the tumor environment such as the vascular growth factor, which has been shown to promote cancer metastasis (8). In addition, the procedures of operative removal of the primary tumor may potentially interfere with the immune system. This adverse effect appears to be mediated through inhibition of the immune activities of CD8 T cells and involves a population of cells in the tumor microenvironment known as myeloid-derived suppressor cells (MDSC) (9). MDSCs contribute to the negative regulation of immune responses under many pathologic conditions, including tumor progression (10). Recent work has suggested that MDSCs can directly infiltrate primary tumor tissues, thereby inducing epithelial-mesenchymal transition (EMT) of tumor cells (11), which may be an essential step for tumor cell dissemination and tumor metastasis (12). This process renders cells both migratory and with invasive properties which are closely correlated with patient survival after operation (13).

In this report, we studied the effect of operative stress on the development of metastases after resection of the primary tumor in mice injected subcutaneously with the murine breast cancer 4T1 cells (14). Our results suggested that the stress of the operative procedure impaired the overall survival and activated the response of MDSCs through the up-regulation of TGFbeta1, VEGF, and IL10 levels. We also evaluated the efficacy of early post-operative MDSCs to determine their role in the development of cancer metastasis.

\section{METHODS}

\section{Cell and Cell Culture Conditions}

The murine breast cell line (4T1) was obtained from American Type Culture Collection (ATCC, Rockville, MD) and grown in RPMI-1640 Medium supplemented with 10\% FBS (Gibco, Eggenstein, Germany), $100 \mathrm{U} / \mathrm{mL}$ penicillin (Sigma, Aldrich, USA), and $100 \mu \mathrm{g} / \mathrm{mL}$ streptomycin (Sigma). All cell lines were maintained at $37^{\circ} \mathrm{C}$, surrounded by a humidified atmosphere of $5 \% \mathrm{CO}_{2}$.

\section{Tumor Tissue Injury Model}

All mouse protocols were approved by the Animal Care and Use Committee of Sichuan University (Chengdu, Sichuan, China). To exclude the potential endocrine differences of mixed mouse sexes, 6-week-old female BALB/c mice (HFK Bioscience, Beijing, China) were used to build the breast tumor model. Approximately $1 \times 10^{6} 4 \mathrm{~T} 1$ freshly prepared tumor cells in the exponential stage of growth were injected subcutaneously into $\mathrm{BALB} / \mathrm{c}$ mice. When the primary tumor diameter reached $4-5 \mathrm{~mm}$ (usually day 6 after inoculation), the mice $(n=120)$ were divided randomly into six equal groups as follows: (1) control group; (2) a contralateral skin incision group involving a $15-20 \mathrm{~mm}$ long skin incision on the contralateral side from the tumor and symmetrical to where the tumor resection was performed; (3) an ipsilateral skin incision group as the control of operative stress to avoid the impact of skin incision on the primary tumors. The skin incision was $15-20 \mathrm{~mm}$ long and 1$3 \mathrm{~mm}$ near the primary tumor, without injury to the tumor itself; (4) a 1/4 tumor tissue removal group; (5) a $3 / 4$ tumor tissue removal group; and (6) a whole tumor removal group. Mice in groups 4, 5, and 6 all had a $15-20 \mathrm{~mm}$ skin incision first and then $1 / 4,3 / 4$, or the entire primary tumors were removed, respectively. Half of the mice of each group $(n=10)$ were used for the survival analysis, while the rest were used for evaluating the number of lung metastases. To ameliorate pain, mice were killed if they exhibited any clinical signs of distress, such as loss of appetite, cachexia, $10 \%$ weight loss, loss of mobility, restlessness, respiratory distress, tumor/skin breakdown, or failure to groom.

\section{Measurement of Lung Metastatic Nodules}

After 28 days, the entire lungs and tumor tissues of mice (three mice from each group) were isolated and weighed. Lungs were fixed in $4 \%$ paraformaldehyde for counting of lung metastases and measurement of the size (diameters) of metastatic nodules using a dissecting microscope. The total number of nodules and the number of nodules over $3 \mathrm{~mm}$ in diameter were also calculated.

\section{Immunohistochemistry}

After 28 days, lung tissues of six groups (three mice from each group) were embedded in Tissue-Tek OCT compound and then frozen in liquid nitrogen. Frozen sections of the primary tumor and lung tissue (all lung cuts of the whole lung tissue, not just the metastases) were used for immunostaining with anti-mouse Gr1 (Abcam, Cambridge, MA USA) and biotinylated goat anti-rat as the secondary antibody (Abcam, Cambridge, MA, USA). An $A B C$ kit and Diaminobenzidine tetrahydrochloride $(\mathrm{DAB})$ were used as a chromogen to visualize antigens.

\section{Isolation of Cells From Primary Tumors and Lung Metastases}

After 28 days, the primary tumors and lung metastases of the rest of the mice (four mice from each group) were collected, cut into small pieces, and incubated at $37^{\circ} \mathrm{C}$ for $2 \mathrm{~h}$ in $20 \mathrm{ml}$ of RPMI (serum-free) medium containing $1 \mathrm{mg} / \mathrm{ml}$ collagenase I (280 U/mg, Gibco) and $2 \mu \mathrm{l}$ of DNase $(2 \mathrm{mg} / \mathrm{ml}$, Sigma). Next, the cell suspension was centrifuged at $300 \mathrm{~g}$ for $10 \mathrm{~min}$. The cells 
were filtered through $40 \mu \mathrm{m}$ nylon filters and centrifuged at $400 \mathrm{~g}$ for $10 \mathrm{~min}$. All living cells were collected from the interface and washed with serum-free RPMI three times.

\section{Flow Cytometry}

Single cells from all six groups were stained with CD11bPerCP-Cy5.5 or PE, Gr1-FTIC, or PE (BD Biosciences, San Jose, CA, USA) for $30 \mathrm{~min}$ at $4^{\circ} \mathrm{C}$. CD $11 \mathrm{~b}^{+} \mathrm{Gr} 1^{+}$MDSCs were stained with antibodies against TGF-beta1, VEGF, and IL-10 (Abcam, Cambridge, MA, USA), with FITC-conjugated secondary antibody labeling for $30 \mathrm{~min}$ at $4^{\circ} \mathrm{C}$. Then the cells were washed three times with serum-free RPMI before being analyzed. For flow cytometry analysis, cells were acquired with an FACSCalibur flow cytometer (BD Biosciences, San Jose, CA, USA) and analyzed with CellQuest software (BD Biosciences, San Jose, CA, USA) as in previous research (15).

\section{ELISA}

The serum levels of TGF-beta1, IL-10, and VEGF of the mice from the $1 / 4$ tumor removal group and the control group were evaluated on the third day after surgery, using corresponding ELISA kits according to the manufacturers' instructions (eBioscience, ThermoFisher Scientific, Waltham, Massachusetts, USA).

\section{Prospective Isolation of MDSC (Gr-1 Positive Cell)}

Gr-1 positive myeloid cells were isolated by magnetic bead sorting (MACS). For this purpose, myeloid cell suspensions from mice thigh bones were prepared according to a previous method (16). Approximately $1 \times 10^{8}$ cells were added to the buffer. Cells were then incubated with an FcR blocking solution for $10 \mathrm{~min}$ at $4^{\circ} \mathrm{C}$ and A Ly6G biotin antibody for $10 \mathrm{~min}$ at $4^{\circ} \mathrm{C}$, and cells were enriched using a technique of magnetic-activated cell sorting (magnetic bead sorting), MDSC isolation kit from Miltenyi Biotec, Bergisch Gladbach, Germany. All procedures were performed according to the manufacturer's instructions (17). The purity of isolated cells was determined by standard flow cytometry analysis using a labeled antibody against mouse Gr-1. The purity of isolated Gr-1 positive cells regularly exceeded $95 \%$.

\section{Co-culture Assays of Epithelial-Mesenchymal Transition (EMT)}

4T1 cells were trypsinized, resuspended in culture media, cocultured with MDSCs, and grew on round cover slips in 24well plates. Trans-wells were incubated at $37^{\circ} \mathrm{C}$ containing $1: 1,1: 3,1: 5,1: 10$, and 1:20 cell number ratio of pre-isolated MDSC cells for $96 \mathrm{~h}$. The morphology of $4 \mathrm{~T} 1$ cells co-cultured with MDSCs for 24, 48, and $96 \mathrm{~h}$ were observed under white light microscope. To explore indirectly the role of MDSCs on EMT of the primary tumor cells, MDSCs were loaded into the upper chambers of a 24 well trans-well insert. After removing non-migrating cells using cotton swabs, inserts were washed in PBS, fixed in 4\% PFA (Paraformaldehyde) and prepared for the immunofluorescence assay. Then the cells were permeabilized with $0.1 \%$ Triton X-100 in PBS, and stained with mouse monoclonal antibodies directed against E-cadherin and Vimentin (Abcam). The secondary antibodies were Alexa Fluor 488-conjugated donkey-anti-rabbit antibody (Invitrogen, 1:500). DAPI (4',6-diamidino-2-phenylindole) $(5 \mu \mathrm{g} / \mathrm{ml})$ was also stained for $10 \mathrm{~min}$. The images were visualized through a Leica TCS SP5 confocal microscope.

\section{Model of Post-operative Gr-1 Clearance}

Approximately $1 \times 10^{6} 4 \mathrm{~T} 1$ freshly prepared tumor cells at their exponential stage of growth were injected subcutaneously into BALB/c female mice. Then $0.15 \mathrm{mg}$ of anti Gr-1 antibody (BD Biosciences) was injected intraperitoneally at the 7th, 12th, and 18th days after inoculation into mice as per the previous procedure (18). We divided the mice into four groups (10 mice for each group) at the 14th day after inoculation: (1) control group; (2) the operation group with $1 / 4$ tumor tissue removed; (3) the Gr-1 clearance group with intraperitoneal injection of Gr1 antibody; (4) the combinational treatment group that receives operation and Gr-1 antibody. The tumor tissue removal groups and tumor tissue removal with Gr-1 clearance group received the respective operation on the 14 th day after the inoculation. Finally, the lung metastases were analyzed to detect the effect of the anti Gr-1 antibody after 28 days.

\section{Statistical Analysis}

Survival times of mice are recorded as mean \pm SEM (standard error of mean), and other data are expressed as the mean $\pm \mathrm{SD}$ (standard deviation). Statistical significance of the difference as for the number of lung metastases and survival between groups was evaluated with independent-sample Student's $t$-test followed by one-way ANOVA (analysis of variance), using SPSS 17.0. $\mathrm{X}^{2}$ test for proportion was used to analyze ELISA and flow cytometry results. Differences were considered significant at $P<0.05$.

\section{RESULTS}

\section{Stress of Operative Removal Impairs the Survival and Promotes Lung Metastasis}

To investigate the effects of surgical stress on mice in this model of breast cancer, we evaluated the status of pulmonary metastases and the survival of the six groups of mice after different procedures of operative intervention: mice with contralateral skin incision, mice with ipsilateral skin incision, 1/4 tumor resection, 3/4 tumor resection, and whole tumor removal. $\mathrm{BALB} / \mathrm{c}$ mice were inoculated with $4 \mathrm{~T} 1$ tumor cells, and primary tumors were removed operatively from mice 10 days later (Figure 1A). The survival outcomes of these 60 mice (10 from each group) were recorded after the initial procedure (day 0 was the inoculation date) and pulmonary metastases of the other 60 mice (10 from each group) were quantified on day 28 according to previously reported method (19).

As indicated in Figure 1B, all groups exhibited tumor metastases during the experimental period. Mice in the control group exhibited a median of $5 \pm 2$ metastases (ranging from 2 to 8 ), while the number of metastatic nodules was $11 \pm 6$ (ranging from 7 to 24) for the contralateral skin incision group, $14 \pm 6$ (ranging from 8 to 23) for ipsilateral skin incision group, $34 \pm 18$ (ranging from 12 to 63 ) for mice with $1 / 4$ 
A

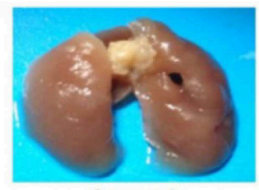

Group 1

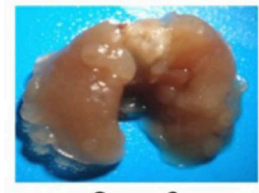

Group 3

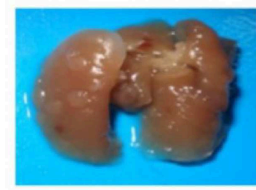

Group 5

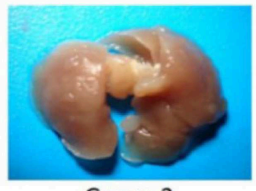

Group 2

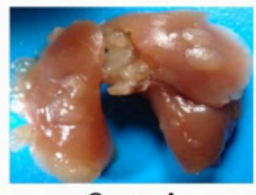

Group 4

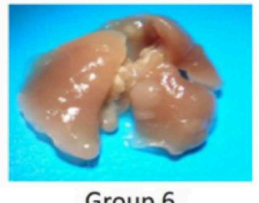

Group 6
B
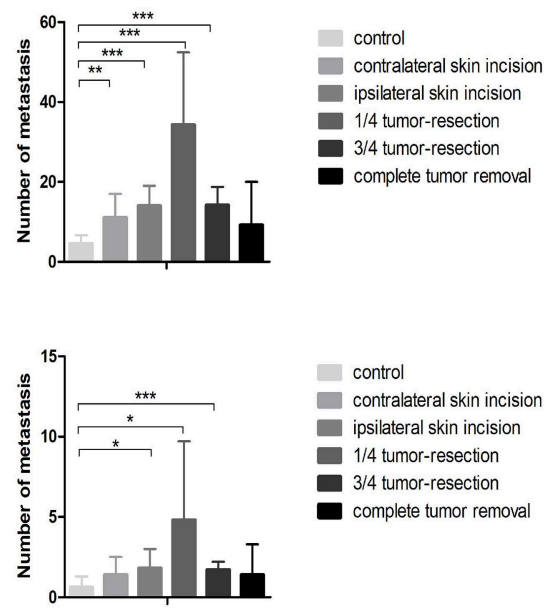

C

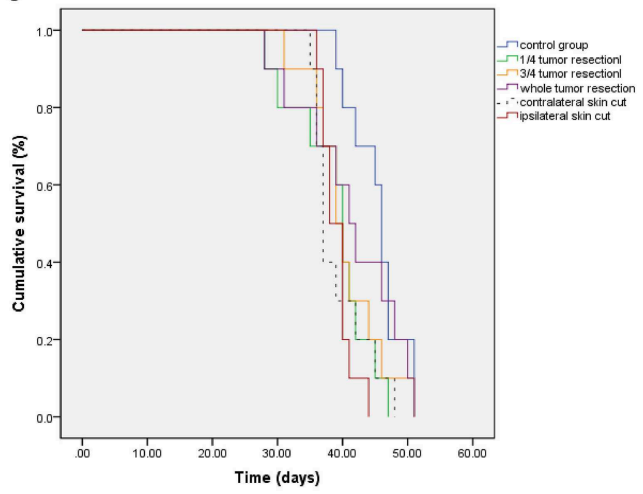

D

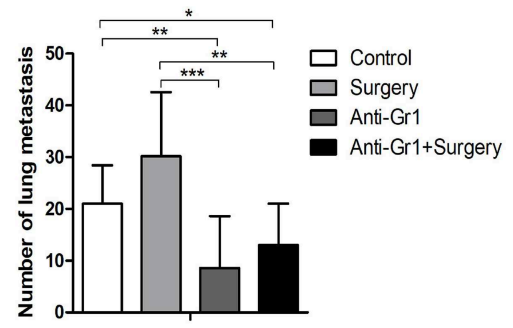

FIGURE 1 | (A) Pulmonary metastases of the mice killed on the 28th day after inoculation. Group 1: control group; 2: contralateral skin incision group; 3: ipsilateral skin incision group; 4: 1/4 tumor resection group; 5: 3/4 tumor resection group (resect a large part of the tumor, simulating the effect of subtotal resection on tumor metastasis and tumor microenvironment); 6: whole tumor resection group; (B) Quantification of pulmonary metastasis: (i) Total number of metastatic nodules; (ii) The number of metastatic nodules of diameters $\geq 3 \mathrm{~mm}$; (C) The survival outcomes of the six groups $(n=10)$ recorded 2 days after the initial procedure (day 0 is inoculated date); (D) The quantification of pulmonary metastases in each group to study the effect of anti-Gr1 treatment on lung metastasis. Mice were divided into four groups: the control group, surgery group (1/4 tumor resection), anti-Gr1 group (The clearance of MDSCs was achieved with anti-Gr1 antibody three times), and anti-Gr1 ${ }^{+}$surgery (1/4 tumor resection) group. Mice went through surgical removal of tumors on the 10th day after inoculation and were sacrificed on the 28th day for the quantification of lung metastasis. ${ }^{*} p<0.05,{ }^{* *} p<0.01$, and ${ }^{* * *} p<0.001$.

tumor removal group, $14 \pm 5$ metastases (ranging from 10 to 25 ) for mice with $3 / 4$ tumor removal group, and $9 \pm 11$ metastases (ranging from 1 to 30 ) for the group with whole tumor resection. All operational groups showed an increased number of metastatic nodules compared with control $(P<0.05)$. Mice with 1/4 tumor resection exhibited the greatest increase in lung metastases as compared with other groups. Metastatic nodules with diameters of more than $3 \mathrm{~mm}$ were considered to be large metastatic nodules. No differences in the incidence of large metastatic nodules were observed between the six groups. The whole tumor resection group failed to demonstrate significantly more metastatic nodules or higher levels of large pulmonary nodules than the control group. Taken together, these results showed that the stress of operation could promote breast cancer metastases. To determine whether primary tumor resection had an effect on survival, we compared the overall survival of mice ( $n$ $=10$ ). The post-inoculation survival time was $44 \pm 2$ days for the control group, $39 \pm 1$ for the contralateral skin incision group, $39 \pm 1$ for the ipsilateral skin incision group, $40 \pm 2$ for the $1 / 4$ tumor removal group, $40 \pm 2$ for the $3 / 4$ tumor removal group, and $42 \pm 3$ for the whole tumor removal group (Figure 1C).

\section{Post-operative Depletion of MDSC Decreases Lung Metastasis After Surgery}

To systematically verify our hypothesis that MDSCs promote breast cancer metastasis, the anti Gr-1 antibody that specifically targeted $\mathrm{Gr}^{+}$MDSCs in tumor-bearing mice was utilized for the depletion of MDSCs according to previous procedure (18). 
The anti Gr-1 antibody was used to deplete MDSCs on the 7 th, 12th, and 18th days after tumor inoculations. The control group and the Gr-1 clearance group did not receive any operative intervention, whereas the tumor tissue removal group and tumor tissue removal with Gr-1 clearance group received the operations at the 14th day after tumor inoculations. Compared with the control group which developed $21 \pm 7$ lung metastases, the whole tumor removal group developed $30 \pm 12$ lung metastases, but failed to demonstrate statistical significance $(P=0.059)$. The Gr-1 clearance groups demonstrated a significant decrease $(P=0.006)$ in the number of metastases, with $9 \pm 10$ for the anti-Gr1 group and $13 \pm 8$ for the surgery plus anti-Gr1 group (Figure 1D). These differences in pulmonary metastases suggested that the depletion of MDSCs with the anti Gr-1 antibody reduced post-operative metastasis.

\section{Stress of Operative Removal Promotes Recruitment of MDSC Into Lung and Tumor Tissues}

To investigate the role of operative procedures on the tumor microenvironment, the primary tumor and lung metastases of 4T1 tumor bearing mice (three mice of each group) were collected for flow cytometry analyses. $\mathrm{CD}_{11 \mathrm{~b}}{ }^{+} \mathrm{Gr} 1^{+}$MDSC cells accounted for $14 \pm 4 \%$ of cells in the primary tumor tissues of the control group, $20 \pm 3 \%$ in contralateral skin incision group, $17 \pm 3 \%$ in ipsilateral skin incision group, $18 \pm 3 \%$ in $1 / 4$ tumor removal group, and $26 \pm 6 \%$ in $3 / 4$ tumor removal group. The

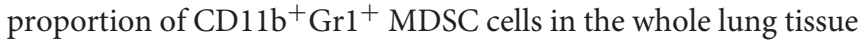
were: $15 \pm 1 \%$ for the control group, $23 \pm 3 \%$ for the contralateral skin incision group, $27 \pm 2 \%$ for the ipsilateral skin incision group, $20 \pm 4 \%$ for the $1 / 4$ tumor removal group, and $23 \pm 3 \%$ for the $3 / 4$ tumor removal group, and $12 \pm 4 \%$ for the whole tumor resection group (Figures $\mathbf{2 A , B}$ ). In addition, we also observed the recruitment of $\mathrm{Grl}^{+}$cells into lung metastases of postoperation mice with immunohistochemistry (Figure 2C). The $1 / 4$ tumor resection group demonstrated the highest number of metastasis, followed by the $3 / 4$ tumor resection group, ipsilateral skin incision group and contralateral skin incision group. The control group and the whole tumor removal group both exhibited limited visible metastases compared with other groups.

\section{Recruited MDSC Induce the Epithelial Mesenchymal Transition (EMT) of Tumor Cells in vitro}

Epithelial Mesenchymal Transition (EMT) theory has been widely acknowledged in explaining tumor metastasis. Purified MDSCs were extracted with magnetic-activated cell sorting (MACS) (Figure 3A). In order to investigate the influence of MDSCs on tumor cell EMT, purified MDSCs (purity of $>90 \%$ ) and tumor cells were co-cultured directly and indirectly. After $96 \mathrm{~h}$ of direct co-culture, we noted that with the gradient concentration of MDSCs in the culture, the proportion of Vimentin positive tumor cells increased. Vimentin positive cells accounted for approximately $4 \pm 2 \%$ of all cells in the control culture, $5 \pm 1 \%$ in culture with $4 \mathrm{~T} 1 \mathrm{:MDSC}=1: 1,8 \pm 2 \%$ in culture with $4 \mathrm{~T} 1: \mathrm{MDSC}=1: 3,9 \pm 2 \%$ in culture with $4 \mathrm{~T} 1 \mathrm{MDSC}$
$=1: 5,14 \pm 3 \%$ in culture with $4 \mathrm{~T} 1: \mathrm{MDSC}=1: 10$, and $14 \pm 3 \%$ in culture with 4T1:MDSC $=1: 20$ (Figure 3B). After 24, 48, and $96 \mathrm{~h}$, we observed an obvious change in the shape and structure of $4 \mathrm{~T} 1$ cells in co-culture with MDSCs compared with those in the control group. Tumor cells gradually grew longer and thinner with their pseudopodia growing until the intercellular junctions disappeared (Figure 3C). Furthermore, tumor cells showed high expression of Vimentin on the cell surface as measured by Western blot assay, while the expression of E-Cadherin decreased markedly (Figure 3D). To explore indirectly the role of MDSCs on EMT of the primary tumor cells, TGF-betal was then added to the $4 \mathrm{~T} 1$ cell cultures as a positive control. EMT could be observed in the culture of the TGF-betal group and MDSC group by immunofluorescence (Figure 3E).

\section{Membrane-Bound Cytokines on MDSCs Appear to Be Critical for EMT in vivo}

To further explore the potential mechanisms through which MDSCs affect the primary tumor microenvironment, MDSCs were extracted from mice after different operational procedures and analyzed by three-color flow cytometry for the expression of TGF-beta1, VEGF, and IL-10. TGF-beta1, VEGF, and IL-10 have been shown to be important cytokines inducing EMT. Through an overlay technique, there was an obvious right shift of the TGF-beta1, VEGF, and IL-10 expressions in primary tumors of mice with operations. In other words, the expression of TGFbeta1, VEGF, and IL-10 on $\mathrm{CD}_{11 \mathrm{~b}}{ }^{+} \mathrm{Gr} 1^{+} \mathrm{MDSC}$ was increased in primary tumor tissues (Figure 4A).

\section{Increased Serum Levels of TGF-Beta 1, VEGF, and IL10}

Serum samples of mice exposed to operative stress in the control group and 1/4 tumor resection group were assayed with ELISA to detect the serum levels of TGF-beta1, VEGF, and IL-10. The concentration of these three cytokines was detected (three mice from each group). In the surgery group (1/4 tumor resection), the levels were as follows: VEGF: $102.9 \pm 10.5 \mathrm{pg} / \mathrm{ml}$, TGF-betal: $75.7 \pm 14.5 \mathrm{pg} / \mathrm{ml}$, and IL-10: $36.0 \pm 3.7 \mathrm{pg} / \mathrm{ml}$; in contrast in control group, VEGF: $52.9 \pm 5.3 \mathrm{pg} / \mathrm{ml}$, TGF-beta1: $31.5 \pm$ $12.2 \mathrm{pg} / \mathrm{ml}$, and IL-10: $15.4 \pm 3.8 \mathrm{pg} / \mathrm{ml}$ (Figure 4B). The serum expression of TGF-beta $1, \mathrm{VEGF}$, and IL-10 increased significantly after the stress of surgery compared with untreated controls.

\section{DISCUSSION}

Breast cancer is one of the most common tumor types and main causes of cancer-related mortality in women worldwide (20). Operative resection of the primary tumor is considered the initial treatment for early-stage, operable breast cancers (21), because the appropriate operation helps remove areas of necrotic tumors that are inaccessible to therapeutic drugs (22) and increases the efficacy of adjuvant treatments in cancer patients. Other positive effects of primary tumor resection include the restoration of the patients' immune system, improvement of the patient's nutritional status, and the control of further metastatic spread (23). Our study was designed to explore these differences and 

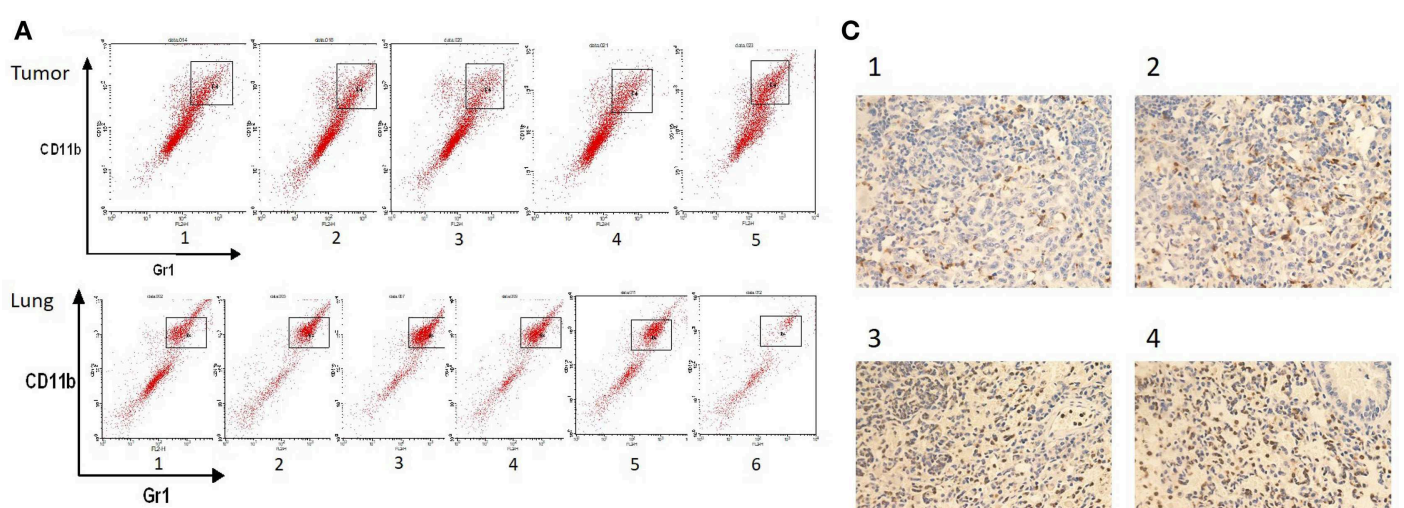

B
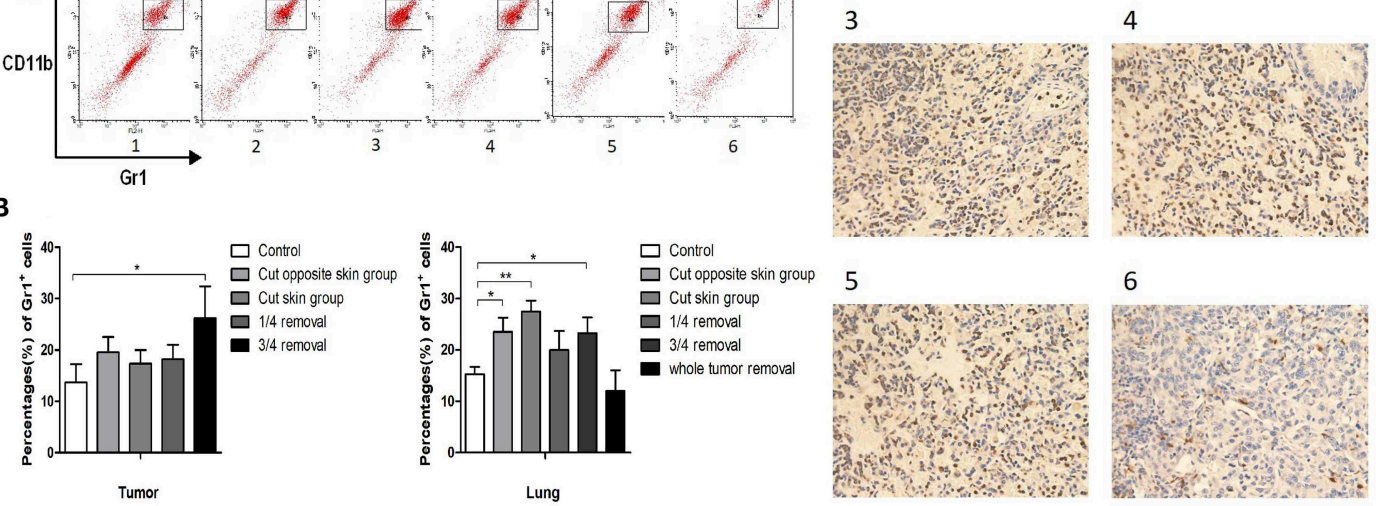

FIGURE 2 | (A) Cells in tumor and lung tissues were stained with antibodies against CD11b and Gr1 and CD11b+ Gr1+ MDSCs were collected for flow cytometry: Group 1: control group; 2: contralateral skin incision group; 3: ipsilateral skin incision group; 4: 1/4 tumor resection group; 5: 3/4 tumor resection group (resect a large part of the tumor, simulating the effect of subtotal resection on tumor metastasis and tumor microenvironment); 6. whole tumor resection group; (B) Quantification of

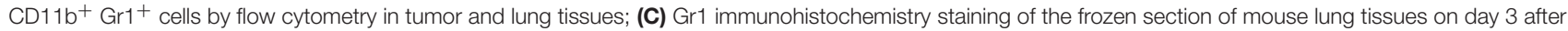
surgery: (1) control group; (2) contralateral skin incision group; (3) ipsilateral skin incision group; (4) 1/4 tumor resection group; (5) 3/4 tumor resection group; (6) whole tumor resection group. ${ }^{*} p<0.05,{ }^{* *} p<0.01$

A

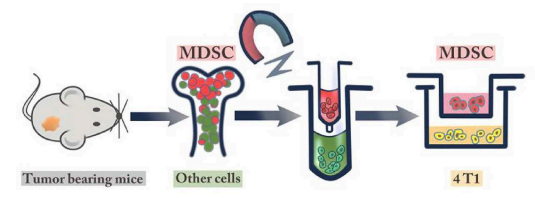

C

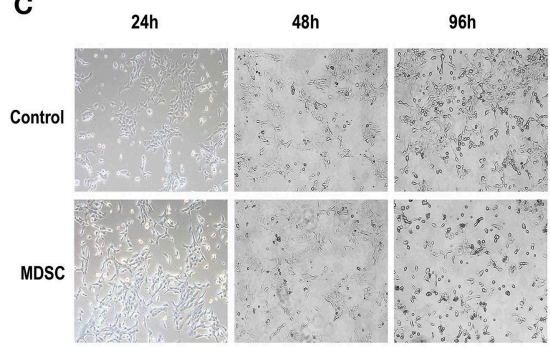

D

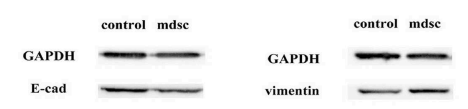

B

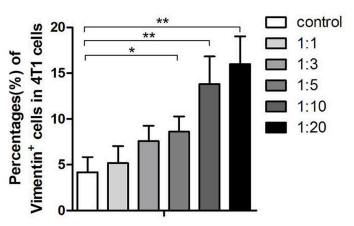

E

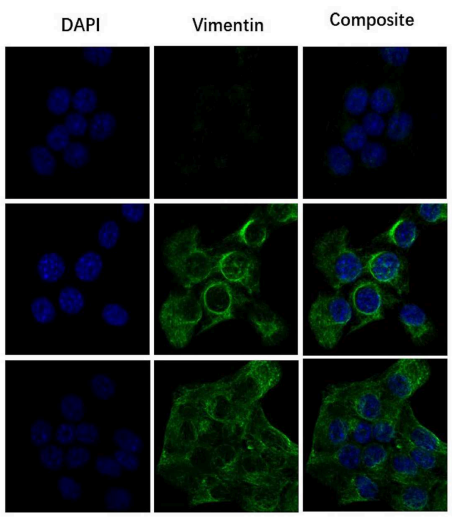

FIGURE 3 | (A) Purified MDSCs were extracted with magnetic-activated cell sorting (MACS); (B) The quantification of Vimentin-positive subsets in 4T1 cells with the gradient elevation of MDSC concentration in direct co-culture; (C). The morphology of 4T1 cells in control buffer and direct co-culture with MDSCs at the time indicated (24, 48, and 96 h); (D) Immunoblots of E-cadherin and vimentin of 4T1 cells in direct co-culture with MDSCs; (E) The immunofluorescence of the DAPI/vimentin positive 4T1 cells indirectly co-cultured with control buffer, TGF- $\beta$ buffer, and MDSCs. Blue, DAPI (nuclear stain); green, Vimentin (nuclear stain). TGF-beta was added as a positive control for induction of EMT. ${ }^{*} p<0.05,{ }^{* *} p<0.01$. 


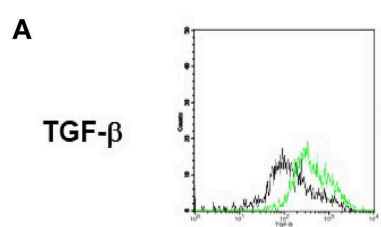

A

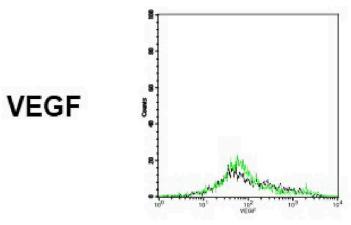

A

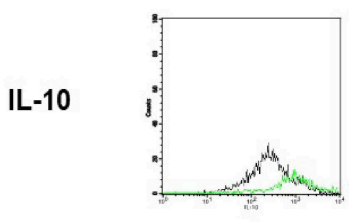

A

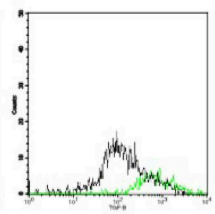

B

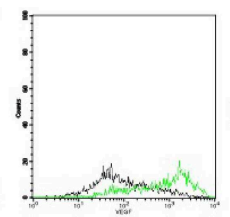

B

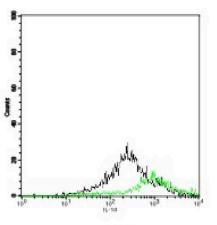

B

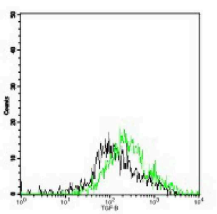

C

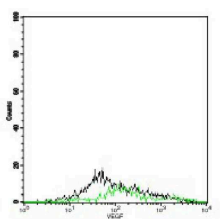

C

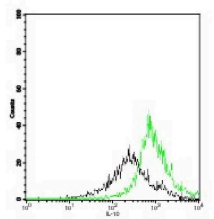

C

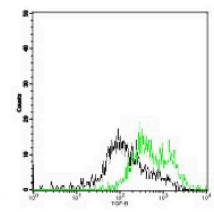

D

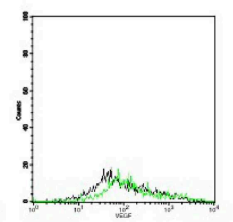

D

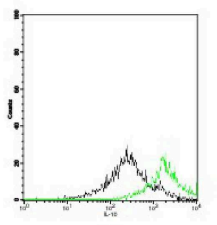

D

B
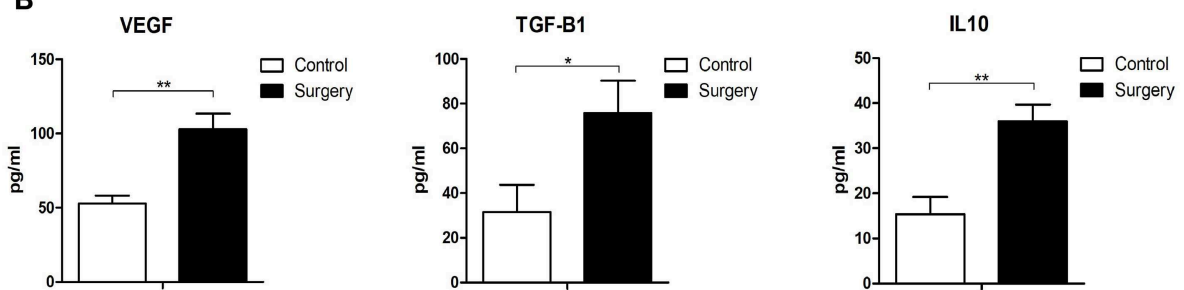

FIGURE 4 | (A) MDSCs were extracted from mouse tumor tissues after different operational procedures and analyzed by three-color flow cytometry for the expression of TGF-beta1, VEGF, and IL-10. The control group (black lines) vs. 1/4 tumor resection group (green lines): A: control group vs. contralateral skin incision group; B: control group vs. ipsilateral skin incision group; C: control group vs. 1/4 tumor resection group; D: control group vs. 3/4 tumor resection group; (B) The serum expression of TGF- $\beta$, VEGF, and IL-10 in the control group and $1 / 4$ tumor resection group on day 3 after operations. ${ }^{*} p<0.05,{ }^{* *} p<0.01$.

to shed light on the role of surgical stress on metastases in mice with breast cancer. The results suggest that the stress of operative removal changes the microenvironment in the primary tumor mass and the areas of lung metastases, which might be the one key determinant of patient survival and distant metastases.

On the other hand, some researchers have reported the negative effects of surgical stress on the growth kinetics of metastases (24). Operative resection of the primary tumor decreases the inhibition of tumor-induced angiogenesis, potentially leading to increased growth of metastases $(25,26)$. As early as the 1910s, studies have reported that implanted tumors rarely developed spontaneous metastases, unless the primary implant was incompletely removed (27). The results were further detailed by a study that incomplete primary tumor resection could lead to larger metastases in mice than in control groups (28). Similar findings were later reported that significant increases in the number of micro-metastases and total lung weight were detected in mice 13 days after surgical removal of primary tumors (29). Moreover, earlier work by Dr. Folkman and his team suggested that the removal of a primary tumor in mice could lead to the exponential growth of its lung metastases (30), and that before resection, the presence of a primary tumor was correlated with an increased level of angiostatin. The operative stress was associated with worse survival in the mouse model, considering tumor volume might be an important factor $(14,26)$. In the clinical setting, previous reports identified no better outcome in infants undergoing primary tumor resection than those without surgical treatment (31). In our experiment, we hypothesized that the increased lung metastases were caused by the stress of the operative procedure that recruited MDSCs to the primary tumor. MDSCs promoted EMT and the release of tumor cells into the circulation, thereby increasing the presence of distant metastasis. Our hypothesis is presented in Figure 5.

The microenvironment related to the tumor itself is believed to represent a major impediment to the therapeutic effectiveness of many cancer treatments (32). MDSCs are immature myeloid cells that can act as immunosuppressive agents and are increased in patients with malignancies, as well as in mouse tumor models (33). It has been reported that the increase of MDSCs in the spleen could be prevented by primary tumor resection (34). 


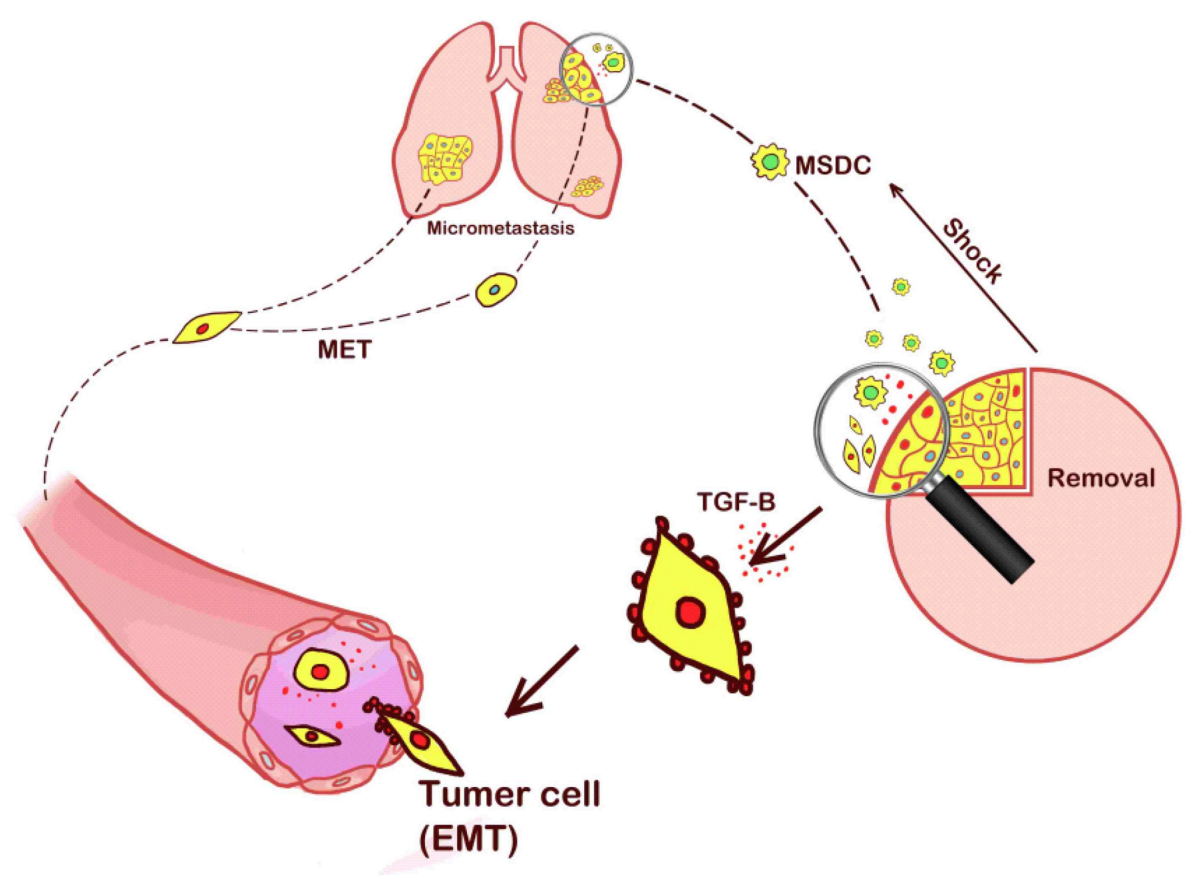

FIGURE 5 | Our hypothesis that the increased lung metastases were caused by the stress of the operative procedure that recruited MDSCs to the primary tumor sites. MDSCs promoted EMT and the release of tumor cells into the circulation, thereby increasing the presence of distant metastasis.

The suppression of antitumor immunity by MDSCs has been attributed to various mechanisms, including interference with $\mathrm{T}$ cell activation and the development of dendritic cells. Different operative procedures can affect $\mathrm{T}$ cell function in the tumor microenvironment (35) by directly disrupting the function of $\mathrm{CD}^{+}$and $\mathrm{CD}^{+} \mathrm{T}$ cells $(10,36)$. In this regard, MDSCs can inhibit immune responses and at the same time promote immune escape of tumor cells leading to metastases $(37,38)$. Several studies have suggested that inhibition of the surgery-induced dysfunction of NK cells can help to prevent postoperative metastasis $(39,40)$. MDSCs appear to be able to suppress innate immunity through down-regulation of NK cell function (41), possibly by inhibiting NK cell cytotoxicity, NKG2D expression, and interferon production through membrane-bound TGF beta1 (41). In this mouse model of breast cancer, we found that MDSCs were rapidly recruited to the site of the primary tumors, suggesting that the acute stress after surgery was associated with the release of MDSCs rather quickly in the postoperative period. Our results supported similar findings with previous literature that the increased populations of MDSCs were related to the development of lung metastasis $(42,43)$. However, the exact association between MDSCs and increased tumor cell metastases still remains unclear. One potentially important finding was that the TGF-beta family could lead to EMT in the primary tumor which increased the metastasis by promoting MDSC infiltration into the tumor microenvironment (44). EMT appears to be activated during cancer invasion and metastasis. This biologic process allows a polarized epithelial cell, which normally interacts with the basement membrane via its basal surface, to undergo multiple biochemical changes that enable it to assume the phenotype of a mesenchymal cell. In our in vitro study, we found that the EMT of tumor cells was induced and that the portion of cells undergoing EMT increased with increasing proportion of MDSCs. Previous reports also confirmed that the up-regulation of TGF-beta family genes could mediate EMT (45-47).

In the in vivo arm of our study, we detected increased tissue and serum levels of TGF-beta 1 in mice with lung metastases after the different operative procedures. TGF-beta can be produced by MDSCs as a suppressive cytokine that inhibits antitumor immune responses (41), which in turn promotes tumor progression by recruiting MDSCs to the tumor microenvironment (44). The up-regulated expression and serum levels of TGF-beta have been reported to indicate the advanced stage, metastasis, and worse prognosis in cancers such as lung cancer (48). We also detected the increased proportion of MDSC subgroups with high expressions of membrane-bound TGF-beta 1, VEGF, and IL-10, both in residual tumor tissues and in lung metastases after operations. A previous study suggested that VEGF contributed to tumor progression, which was positively correlated with MDSC levels in cancer patients (49). The upregulation of VEGF on MDSCs of primary tumors could in turn promote the activation of the MDSCs and therefore, might be a potentially useful target to manipulate MDSC expansion (10). Angiogenesis is not only a key stimulator for the growth of the primary tumor, but also important for the expansion of established metastases. Angiostatin, a plasminogen fragment characterized with antiangiogenic effects, was able to suppress metastasis growth in vivo by inhibiting tumor angiogenesis (30). They also found that as early as 5 days after primary tumor resection, lung metastases were 
soon infiltrated with endothelial cells, indicating that tumor metastases were associated with the formation of blood vessels (29). IL-10 can be expressed by MDSCs as a stimulator for tumor progression $(50,51)$. High serum levels of IL-10 have previously been detected in patients with ovarian cancer (5254 ), and are consistently associated with advanced progression and poor prognosis in multiple cancer types (55-57). A more

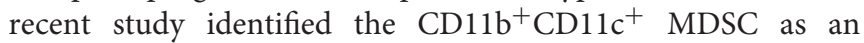
important IL-10-producer in tumor microenvironments which helps to establish a friendly environment for tumor growth (58). Moreover, selectively blocking the IL-10 signaling is an effective therapeutic strategy which potently inhibits MDSC activities (58). These results suggested that operations could promote the recruitment of MDSCs to residual primary tumors as well as lung metastases, and is associated with increased expression and release of cytokines such as TGF-beta 1, VEGF, and IL-10 by MDSCs. Those cytokines, together with increased MDSCs, can be a possible explanation for the increased metastases after operations.

MDSCs have been considered as potential effectors of cancer immune evasion and can enhance tumor-induced immune suppression in the tumor microenvironment $(59,60)$, making it a novel target for therapeutic intervention. Some recent work has suggested the potential of MDSC depletion as a strategy for cancer treatment (61). Targeted depletion of MDSCs in pancreatic cancer increases the intra-tumor accumulation of adaptive immunity and remodels the tumor microenvironment (62). Anti Grl antibody is widely used for depletion of MDSCs (63). In a previous study, we found that si-A20 expression or anti Gr1 antibody treatment could induce MDSC apoptosis and exert an anti-tumor effect (18). The present findings suggest that the

\section{REFERENCES}

1. Coughlin SS, Ekwueme DU. Breast cancer as a global health concern. Cancer Epidemiol. (2009) 33:315-8. doi: 10.1016/j.canep.2009.10.003

2. Scully OJ, Bay BH, Yip G, Yu Y. Breast cancer metastasis. Cancer Genomics Proteomics. (2012) 9:311-20.

3. National Cancer Institute. Breast Cancer (PDQ). Treatment. Stage IIIB, Inoperable IIIC, IV, Recurrent, and Metastatic Breast Cancer. (2007). Available online at: http://www.cancer.gov/cancertopics/pdq/treatment/ breast/HealthProfessional/page8 (accessed January 30, 2007).

4. Veronesi U, Cascinelli N, Mariani L, Greco M, Saccozzi R, Luini A, et al. Twenty-year follow-up of a randomized study comparing breast-conserving surgery with radical mastectomy for early breast cancer. N Engl J Med. (2002) 347:1227-32. doi: 10.1056/NEJMoa020989

5. Singletary SE, Walsh G, Vauthey JN, Curley S, Sawaya R, Weber KL, et al. A role for curative surgery in the treatment of selected patients with metastatic breast cancer. Oncologist. (2003) 8:241-51. doi: 10.1634/theoncologist.8-3-241

6. Ben-Eliyahu S. The promotion of tumor metastasis by surgery and stress: immunological basis and implications for psychoneuroimmunology. Brain Behav Immun. (2003) 17(Suppl 1):S27-36. doi: 10.1016/S0889-1591(02)00063-6

7. Karnoub AE, Dash AB, Vo AP, Sullivan A, Brooks MW, Bell GW, et al. Mesenchymal stem cells within tumour stroma promote breast cancer metastasis. Nature. (2007) 449:557-63. doi: 10.1038/nature06188

8. Cursiefen C, Chen L, Borges LP, Jackson D, Cao J, Radziejewski C, et al. VEGF-A stimulates lymphangiogenesis and hemangiogenesis in depletion of MDSCs by the anti Gr1 antibody decreases postoperative lung metastases. And in the future, the adverse effects of recruited MDSCs, possibly before and also in selected situations after surgery, can be eliminated by the anti Gr1 antibody.

\section{CONCLUSIONS}

The stress of operative procedures promotes MDSC recruitment into the residual primary tumor and lung metastasis. Membranebound TGF-beta 1, VEGF, and IL-10 on MDSCs could induce tumor cells to undergo EMT leading to tumor cell metastases. The MDSC depletion with the anti Gr1 antibody both before as well as at the time of resection of primary tumors provides a promising approach for the prevention of metastases of breast cancer.

\section{DATA AVAILABILITY}

All data generated or analyzed during this study are included in this article.

\section{ETHICS STATEMENT}

All mouse protocols were approved by the Animal Care and Use Committee of Sichuan University (Chengdu, Sichuan, China).

\section{AUTHOR CONTRIBUTIONS}

MW collected, analyzed the data, and wrote the article. XM and XW provided the idea. TY modified the article. YZ edited the pictures. All authors reviewed the manuscript. inflammatory neovascularization via macrophage recruitment. J Clin Invest. (2004) 113:1040-50. doi: 10.1172/JCI200420465

9. Stein U, Arlt F, Walther W, Smith J, Waldman T, Harris ED, et al. The metastasis-associated gene S100A4 is a novel target of beta-catenin/T-cell factor signaling in colon cancer. Gastroenterology. (2006) 131:1486-500. doi: 10.1053/j.gastro.2006.08.041

10. Gabrilovich DI, Nagaraj S. Myeloid-derived suppressor cells as regulators of the immune system. Nat Rev Immunol. (2009) 9:162-74. doi: 10.1038/nri2506

11. Toh B, Wang X, Keeble J, Sim WJ, Khoo K, Wong WC, et al. Mesenchymal transition and dissemination of cancer cells is driven by myeloid-derived suppressor cells infiltrating the primary tumor. PLoS Biol. (2011) 9:e1001162. doi: 10.1371/journal.pbio.1001162

12. Thiery JP. Epithelial-mesenchymal transitions in tumour progression. Nat Rev Cancer. (2002) 2:442-54. doi: 10.1038/nrc822

13. Iwatsuki M, Mimori K, Yokobori T, Ishi H, Beppu T, Nakamori $\mathrm{S}$, et al. Epithelial-mesenchymal transition in cancer development and its clinical significance. Cancer Sci. (2010) 101:293-9. doi: 10.1111/j.1349-7006.2009.01419.x

14. Danna EA, Sinha P, Gilbert M, Clements VK, Pulaski BA, OstrandRosenberg S. Surgical removal of primary tumor reverses tumor-induced immunosuppression despite the presence of metastatic disease. Cancer Res. (2004) 64:2205-11. doi: 10.1158/0008-5472.CAN-03-2646

15. Yin $\mathrm{T}, \mathrm{He} \mathrm{S}$, Liu $\mathrm{X}$, Jiang $\mathrm{W}, \mathrm{Ye} \mathrm{T}$, Lin $\mathrm{Z}$, et al. Extravascular red blood cells and hemoglobin promote tumor growth and therapeutic resistance as endogenous danger signals. J Immunol. (2015) 194:429-37. doi: 10.4049/jimmunol.1400643 
16. Rieber N, Singh A, Oz H, Carevic M, Bouzani M, Amich J, et al. Pathogenic fungi regulate immunity by inducing neutrophilic myeloidderived suppressor cells. Cell Host Microbe. (2015) 17:507-14. doi: 10.1016/j.chom.2015.02.007

17. Oz HH, Zhou B, Voss P, Carevic M, Schroth C, Frey N, et al. Pseudomonas aeruginosa airway infection recruits and modulates neutrophilic myeloid-derived suppressor cells. Front Cell Infect Microbiol. (2016) 6:167. doi: 10.3389/fcimb.2016.00167

18. Shao B, Wei X, Luo M, Yu J, Tong A, Ma X, et al. Inhibition of A20 expression in tumor microenvironment exerts anti-tumor effect through inducing myeloid-derived suppressor cells apoptosis. Sci Rep. (2015) 5:16437. doi: $10.1038 /$ srep 16437

19. Huang X, Wong MK, Yi H, Watkins S, Laird AD, Wolf SF, et al. Combined therapy of local and metastatic $4 \mathrm{~T} 1$ breast tumor in mice using SU6668, an inhibitor of angiogenic receptor tyrosine kinases, and the immunostimulator B7.2-IgG fusion protein. Cancer Res. (2002) 62: 5727-35.

20. Ferlay J, Soerjomataram I, Dikshit R, Eser S, Mathers C, Rebelo M, et al. Cancer incidence and mortality worldwide: sources, methods and major patterns in GLOBOCAN 2012. Int J Cancer. (2015) 136:E359-86. doi: $10.1002 /$ ijc. 29210

21. Chatterjee S, Chaubal R, Maitra A, Gardi N, Dutt A, Gupta S, et al. Pre-operative progesterone benefits operable breast cancer patients by modulating surgical stress. Breast Cancer Res Treat. (2018) 170:431-8. doi: 10.1007/s10549-018-4749-3

22. Dauplat J, Le Bouedec G, Pomel C, Scherer C. Cytoreductive surgery for advanced stages of ovarian cancer. Semin Surg Oncol. (2000) 19:42-8. doi: 10.1002/1098-2388(200007/08)19:1<42::AID-SSU7>3.0.CO;2-M

23. Kusmartsev S, Nefedova Y, Yoder D, Gabrilovich DI. Antigen-specific inhibition of $\mathrm{CD}^{+} \mathrm{T}$ cell response by immature myeloid cells in cancer is mediated by reactive oxygen species. J Immunol. (2004) 172:989-99. doi: 10.4049/jimmunol.172.2.989

24. Fisher ER, Fisher B. Experimental studies of factors influencing development of hepatic metastases. XVII. Role of thyroid. Cancer Res. (1966) 26:2248-54.

25. Retsky M, Bonadonna G, Demicheli R, Folkman J, Hrushesky W, Valagussa P. Hypothesis: induced angiogenesis after surgery in premenopausal nodepositive breast cancer patients is a major underlying reason why adjuvant chemotherapy works particularly well for those patients. Breast Cancer Res. (2004) 6: R372-4. doi: 10.1186/bcr804

26. Retsky M, Demicheli R, Hrushesky W. Pre-menopausal status accelerates relapse in node positive breast cancer: hypothesis links angiogenesis, screening controversy. Breast Cancer Res Treat. (2001) 65:217-24. doi: 10.1023/A:1010626302152

27. Demicheli R, Retsky MW, Hrushesky WJ, Baum M, Gukas ID. The effects of surgery on tumor growth: a century of investigations. Ann Oncol. (2008) 19:1821-8. doi: 10.1093/annonc/mdn386

28. Tyzzer EE. Factors in the production and growth of tumor metastases. J Med Res. (1913) 28:309-332.1.

29. Holmgren L, O'Reilly MS, Folkman J. Dormancy of micrometastases: balanced proliferation and apoptosis in the presence of angiogenesis suppression. Nat Med. (1995) 1:149-53. doi: 10.1038/nm0295-149

30. O'Reilly MS, Holmgren L, Shing Y, Chen C, Rosenthal RA, Moses $\mathrm{M}$, et al. Angiostatin: a novel angiogenesis inhibitor that mediates the suppression of metastases by a Lewis lung carcinoma. Cell. (1994) 79:315-28. doi: 10.1016/0092-8674(94)90200-3

31. Guglielmi M, De Bernardi B, Rizzo A, Federici S, Boglino C, Siracusa F, et al. Resection of primary tumor at diagnosis in stage IV-S neuroblastoma: does it affect the clinical course? J Clin Oncol. (1996) 14:1537-44. doi: 10.1200/JCO.1996.14.5.1537

32. Whiteside TL, The tumor microenvironment and its role in promoting tumor growth. Oncogene. (2008) 27:5904-12. doi: 10.1038/onc.2008.271

33. Gabitass RF, Annels NE, Stocken DD, Pandha HA, Middleton GW. Elevated myeloid-derived suppressor cells in pancreatic, esophageal and gastric cancer are an independent prognostic factor and are associated with significant elevation of the Th2 cytokine interleukin-13. Cancer Immunol Immunother. (2011) 60:1419-30. doi: 10.1007/s00262-011-1028-0

34. Rashid OM, Nagahashi M, Ramachandran S, Graham L, Yamada A, Spiegel $\mathrm{S}$, et al. Resection of the primary tumor improves survival in metastatic breast cancer by reducing overall tumor burden. Surgery. (2013) 153:771-8. doi: 10.1016/j.surg.2013.02.002

35. Allendorf JD, Bessler M, Horvath KD, Marvin MR, Laird DA, Whelan RL. Increased tumor establishment and growth after open vs laparoscopic surgery in mice may be related to differences in postoperative T-cell function. Surg Endosc. (1999) 13:233-5. doi: 10.1007/s004649900952

36. Nagaraj S, Schrum AG, Cho HI, Celis E, Gabrilovich DI. Mechanism of T cell tolerance induced by myeloid-derived suppressor cells. J Immunol. (2010) 184:3106-16. doi: 10.4049/jimmunol.0902661

37. Gallina G, Dolcetti L, Serafini P, De Santo C, Marigo I, Colombo MP, et al. Tumors induce a subset of inflammatory monocytes with immunosuppressive activity on $\mathrm{CD}^{+} \mathrm{T}$ cells. J Clin Invest. (2006) 116:277790. doi: $10.1172 /$ JCI28828

38. Frey AB. Myeloid suppressor cells regulate the adaptive immune response to cancer. J Clin Invest. (2006) 116:2587-90. doi: 10.1172/JCI29906

39. Tai LH, Zhang J, Scott KJ, de Souza CT, Alkayyal AA, Ananth AA, et al. Perioperative influenza vaccination reduces postoperative metastatic disease by reversing surgery-induced dysfunction in natural killer cells. Clin Cancer Res. (2013) 19:5104-15. doi: 10.1158/1078-0432.CCR-13-0246

40. Tai LH, de Souza CT, Belanger S, Ly L, Alkayyal AA, Zhang J, et al. Preventing postoperative metastatic disease by inhibiting surgeryinduced dysfunction in natural killer cells. Cancer Res. (2013) 73:97-107. doi: 10.1158/0008-5472.CAN-12-1993

41. Li H, Han Y, Guo Q, Zhang M, Cao X. Cancer-expanded myeloid-derived suppressor cells induce anergy of NK cells through membrane-bound TGFbeta 1. J Immunol. (2009) 182:240-9. doi: 10.4049/jimmunol.182.1.240

42. Joyce JA, Pollard JW. Microenvironmental regulation of metastasis. Nat Rev Cancer. (2009) 9:239-52. doi: 10.1038/nrc2618

43. Kim K, Skora AD, Li Z, Liu Q, Tam AJ, Blosser RL, et al. Eradication of metastatic mouse cancers resistant to immune checkpoint blockade by suppression of myeloid-derived cells. Proc Natl Acad Sci USA. (2014) 111:11774-9. doi: 10.1073/pnas.1410626111

44. Yang L, Huang J, Ren X, Gorska AE, Chytil A, Aakre M, et al. Abrogation of TGF beta signaling in mammary carcinomas recruits $\mathrm{Gr}-1^{+} \mathrm{CD} 11 \mathrm{~b}^{+}$ myeloid cells that promote metastasis. Cancer Cell. (2008) 13:23-35. doi: 10.1016/j.ccr.2007.12.004

45. Galliher AJ, Schiemann WP. Beta3 integrin and Src facilitate transforming growth factor-beta mediated induction of epithelial-mesenchymal transition in mammary epithelial cells. Breast Cancer Res. (2006) 8:R42. doi: $10.1186 /$ bcr 1524

46. Han G, Lu SL, Li AG, He W, Corless CL, Kulesz-Martin M, et al. Distinct mechanisms of TGF-beta1-mediated epithelial-to-mesenchymal transition and metastasis during skin carcinogenesis. J Clin Invest. (2005) 115:1714-23. doi: 10.1172/JCI24399

47. Derynck R, Akhurst RJ, Balmain A. TGF-beta signaling in tumor suppression and cancer progression. Nature Genet. (2001) 29:117-29. doi: $10.1038 /$ ng 1001-117

48. Lippitz BE. Cytokine patterns in patients with cancer: a systematic review. Lancet Oncol. (2013) 14:e218-28. doi: 10.1016/S1470-2045(12)70582-X

49. Gonda K, Shibata M, Ujiie D, Ashizawa M, Okayama H, Fujita S, et al. Correlation of VEGF with immune suppression involving MDSC, malnutrition, and prognosis in patients with gastric and colorectal cancer. $J$ Clin Oncol. (2018) 36:582. doi: 10.1200/JCO.2018.36.4_suppl.582

50. Hardbower DM, Coburn LA, Asim M, Singh K, Sierra JC, Barry DP, et al. EGFR-mediated macrophage activation promotes colitis-associated tumorigenesis. Oncogene. (2017) 36:3807. doi: 10.1038/onc.2017.23

51. Shvedova AA, Kisin ER, Yanamala N, Tkach AV, Gutkin DW, Star A, et al. MDSC and TGF $\beta$ are required for facilitation of tumor growth in the lungs of mice exposed to carbon nanotubes. Cancer Res. (2015) 75:1615. doi: 10.1158/0008-5472.CAN-14-2376

52. Gotlieb WH, Abrams JS, Watson JM, Velu TJ, Berek JS, Martinez-Maza O. Presence of interleukin 10 (IL-10) in the ascites of patients with ovarian and other intra-abdominal cancers. Cytokine. (1992) 4:385-90. doi: 10.1016/1043-4666(92)90082-3

53. Mustea A, Konsgen D, Braicu EI, Pirvulescu C, Sun P, Sofroni D, et al. Expression of IL-10 in patients with ovarian carcinoma. Anticancer Res. (2006) 26:1715-8. 
54. Santin AD, Bellone S, Ravaggi A, Roman J, Smith CV, Pecorelli S, et al. Increased levels of interleukin-10 and transforming growth factor-beta in the plasma and ascitic fluid of patients with advanced ovarian cancer. BJOG. (2001) 108:804-8. doi: 10.1016/S0306-5456(00) 00206-0

55. Zeni E, Mazzetti L, Miotto D, Lo Cascio N, Maestrelli P, Querzoli $\mathrm{P}$, et al. Macrophage expression of interleukin-10 is a prognostic factor in nonsmall cell lung cancer. Eur Respir J. (2007) 30:627-32. doi: $10.1183 / 09031936.00129306$

56. Nowak M, Glowacka E, Szpakowski M, Szyllo K, Malinowski A, Kulig A, et al. Proinflammatory and immunosuppressive serum, ascites and cyst fluid cytokines in patients with early and advanced ovarian cancer and benign ovarian tumors. Neuro Endocrinol Lett. (2010) 31:375-83.

57. Lech-Maranda E, Bienvenu J, Michallet AS, Houot R, Robak T, Coiffier B, et al. Elevated IL-10 plasma levels correlate with poor prognosis in diffuse large B-cell lymphoma. Eur Cytokine Netw. (2006) 17:60-6.

58. Hart KM, Byrne KT, Molloy MJ, Usherwood EM, Berwin B. IL10 immunomodulation of myeloid cells regulates a murine model of ovarian cancer. Front Immunol. (2011) 2:29. doi: 10.3389/fimmu.2011. 00029

59. Ostrand-Rosenberg S, Sinha P, Beury DW, Clements VK. Cross-talk between myeloid-derived suppressor cells (MDSC), macrophages, and dendritic cells enhances tumor-induced immune suppression. Semin Cancer Biol. (2012) 22:275-81. doi: 10.1016/j.semcancer.2012.01.011

60. Eruslanov E, Kaliberov S, Daurkin I, Kaliberova L, Buchsbaum D, Vieweg J, et al. Altered expression of 15-hydroxyprostaglandin dehydrogenase in tumor-infiltrated CD11b myeloid cells: a mechanism for immune evasion in cancer. J Immunol. (2009) 182:7548-57. doi: 10.4049/jimmunol.0802358

61. Kim YS, Kim YJ, Lee JM, Kim EK, Park YJ, Choe SK, et al. Functional changes in myeloid-derived suppressor cells (MDSCs) during tumor growth: FKBP51 contributes to the regulation of the immunosuppressive function of MDSCs. $J$ Immunol. (2012) 188:4226-34. doi: 10.4049/jimmunol.1103040

62. Stromnes IM, Brockenbrough JS, Izeradjene K, Carlson MA, Cuevas C, Simmons RM, et al. Targeted depletion of an MDSC subset unmasks pancreatic ductal adenocarcinoma to adaptive immunity. Gut. (2014) 63:1769-81. doi: 10.1136/gutjnl-2013-306271

63. Matsuzaki J, Tsuji T, Chamoto K, Takeshima T, Sendo F, Nishimura T. Successful elimination of memory-type $\mathrm{CD}^{+} \mathrm{T}$ cell subsets by the administration of anti-Gr-1 monoclonal antibody in vivo. Cell Immunol. (2003) 224:98-105. doi: 10.1016/j.cellimm.2003.08.009

Conflict of Interest Statement: The authors declare that the research was conducted in the absence of any commercial or financial relationships that could be construed as a potential conflict of interest.

Copyright () 2019 Ma, Wang, Yin, Zhao and Wei. This is an open-access article distributed under the terms of the Creative Commons Attribution License (CC BY). The use, distribution or reproduction in other forums is permitted, provided the original author(s) and the copyright owner(s) are credited and that the original publication in this journal is cited, in accordance with accepted academic practice. No use, distribution or reproduction is permitted which does not comply with these terms. 